\title{
Radiative spacetimes approaching the Vaidya metric
}

\author{
Jiří Podolský and Otakar Svítek \\ Institute of Theoretical Physics, Charles University in Prague, \\ Faculty of Mathematics and Physics, V Holešovičkách 2, \\ 18000 Praha 8, Czech Republic
}

March 2, 2019

\begin{abstract}
We analyze a class of exact type II solutions of the Robinson-Trautman family which contain pure radiation and (possibly) a cosmological constant. It is shown that these spacetimes exist for any sufficiently smooth initial data, and that they approach the spherically symmetric Vaidya-(anti-)de Sitter metric. We also investigate extensions of the metric, and we demonstrate that their order of smoothness is in general only finite. Some applications of the results are outlined.
\end{abstract}

PACS: 04.30.-w, 04.20.Jb, 04.20.Ex

\section{Introduction}

The classic Vaidya metric [1-4] (see also [5,6] followed by reprints of the original Vaidya papers) is a spherically symmetric type D solution of the Einstein equations in the presence of pure radiation matter field which propagates at the speed of light. In various contexts this "null dust" may be interpreted as high-frequency electromagnetic or gravitational waves, incoherent superposition of aligned waves with random phases and polarisations, or as massless scalar particles or neutrinos. The Vaidya solution depends on an arbitrary "mass function" $m(u)$ of the retarded time $u$ which characterises the profile of the pure radiation (it is a "retarded mass" measured at conformal infinity). Various sandwiches and shells of null matter can thus be constructed that are bounded either by flat $(m=0)$ or Schwarzschild-like $(m=$ const $\neq 0)$ vacuum regions. Due to this property such solutions have been extensively used as models of spherically symmetric gravitational collapse of a star, as an exterior solution describing objects consisting of heat-conducting matter, as an interesting toy model for investigation of singularities and their possible removal by quantum effects, for studies of various formulations of the cosmic censorship conjecture on both classical and quantum level, process of black-hole evaporation, and for other purposes (see, e.g., [7-16] for more details and related references). 
In fact, the Vaidya spacetime belongs to a large Robinson-Trautman class of expanding nontwisting solutions $[4,17,18]$. Various aspects of this family have been studied in the last two decades. In particular, the existence, asymptotic behaviour and global structure of vacuum Robinson-Trautman spacetimes of type II with spherical topology were investigated [19-28], most recently in the works of Chruściel and Singleton [29-31]. In these rigorous studies, which were based on the analysis of solutions to the nonlinear RobinsonTrautman equation for generic, arbitrarily strong smooth initial data, the spacetimes were shown to exist globally for all positive retarded times, and to converge asymptotically to a corresponding Schwarzschild metric. Interestingly, extension across the "Schwarzschildlike" event horizon can only be made with a finite order of smoothness. Subsequently, these results were generalized in $[32,33]$ to the Robinson-Trautman vacuum spacetimes which admit a nonvanishing cosmological constant $\Lambda$. It was demonstrated that these cosmological solutions settle down exponentially fast to a Schwarzschild-(anti-)de Sitter solution at large times $u$. In certain cases the interior of a Schwarzschild-de Sitter black hole can be joined to an "external" cosmological Robinson-Trautman region across the horizon with a higher order of smoothness than in the corresponding case with $\Lambda=0$. For the extreme value $9 \Lambda m^{2}=1$, the extension is smooth but not analytic (and not unique). The models with $\Lambda>0$ also exhibit explicitly the cosmic no-hair conjecture under the presence of gravitational waves. On the other hand, when $\Lambda<0$ the smoothness of such an extension is lower.

Our aim here is to further extend the Chruściel-Singleton analysis of the RobinsonTrautman vacuum equation by including matter, namely pure radiation. It was argued already by Bičák and Perjés [34] that with $\Lambda=0$ such spacetimes should generically approach the Vaidya metric asymptotically. We will analyze this problem in more detail, including also the possibility of $\Lambda \neq 0$ in which case the Robinson-Trautman spacetimes containing pure radiation can be shown to approach the radiating Vaidya-(anti-)de Sitter metric.

\section{The metric and field equations}

In standard coordinates the Robinson-Trautman metric has the form $[4,18,35]$

$$
\mathrm{d} s^{2}=-\left(K-2 r(\ln P)_{, u}-2 \frac{m}{r}-\frac{\Lambda}{3} r^{2}\right) \mathrm{d} u^{2}-2 \mathrm{~d} u \mathrm{~d} r+2 \frac{r^{2}}{P^{2}} \mathrm{~d} \zeta \mathrm{d} \bar{\zeta},
$$

where $K=\Delta(\ln P)$ with $\Delta \equiv 2 P^{2} \partial_{\zeta} \partial_{\bar{\zeta}}$ being the Gaussian curvature of the 2 -surfaces $2 P^{-2} \mathrm{~d} \zeta \mathrm{d} \bar{\zeta}, m(u)$ is the mass function, and $\Lambda$ is the cosmological constant. When the function $P(u, \zeta, \bar{\zeta})$ satisfies the fourth-order Robinson-Trautman field equation

$$
\Delta K+12 m(\ln P)_{, u}-4 m_{, u}=2 \kappa n^{2},
$$

the metric describes a spacetime (generally of the Petrov type II) filled with pure radiation field $T_{\mu \nu}=n^{2}(u, \zeta, \bar{\zeta}) r^{-2} k_{\mu} k_{\nu}$, where $\mathbf{k}=\partial_{r}$ is aligned along the degenerate principal null direction (we use the convention $G_{\mu \nu}+\Lambda g_{\mu \nu}=\kappa T_{\mu \nu}$ ). In particular, vacuum RobinsonTrautman spacetimes are given by $n=0$, in which case $m$ can be set to a constant by a suitable coordinate transformation [4]. Vacuum spacetimes (11) — possibly with a nonvanishing $\Lambda$ - thus satisfy the equation $12 m(\ln P)_{, u}=-\Delta K$. These include the spherically symmetric Schwarzschild-(anti-)de Sitter solution which corresponds to $P_{0}=1+\frac{1}{2} \zeta \bar{\zeta}$. Indeed, replacing the complex stereographic coordinate $\zeta$ with angular coordinates by $\zeta=\sqrt{2} e^{i \phi} \tan (\theta / 2)$, we obtain $2 P_{0}^{-2} \mathrm{~d} \zeta \mathrm{d} \bar{\zeta}=\mathrm{d} \theta^{2}+\sin ^{2} \theta \mathrm{d} \phi^{2}$, and $K_{0}=\Delta_{0} \ln \left(P_{0}\right)=1$. 

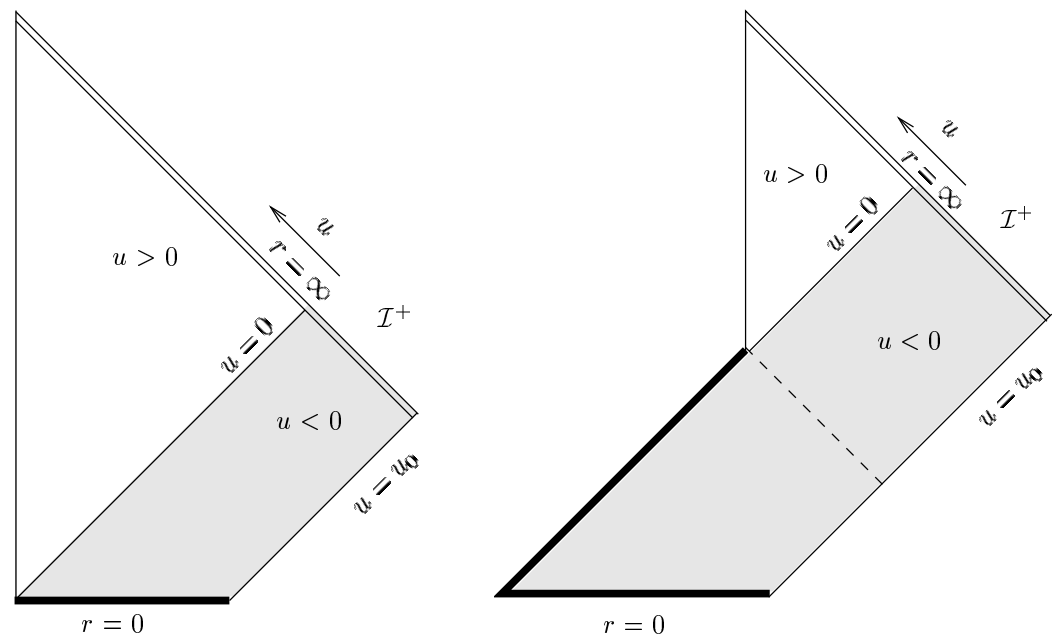

Figure 1: Schematic conformal diagrams of the Robinson-Trautman exact spacetimes which exist for any smooth initial data prescribed on $u_{0}$. Pure radiation field is present in the shaded region $u<0$. Near $u=0$ the solutions approach the Vaidya metric, and can be extended to flat Minkowski region $u>0$. Thick line indicates the curvature singularity at $r=0$ whereas double line represents future conformal infinity $\mathcal{I}^{+}$at $r=\infty(\Lambda=0$ is assumed). The global structure depends on the value of the parameter $\mu$ of the linear mass function (8): left diagram corresponds to $\mu>1 / 16$, the right one applies when $\mu \leq 1 / 16$.

Here we will restrict ourselves to nonvacuum cases for which the dependence of the mass function $m(u)$ on the null coordinate $u$ is only caused by a homogeneous pure radiation with the density $n^{2}(u) r^{-2}$. When the mass function $m(u)$ is decreasing, the field equation (2) can be naturally split into the following pair,

$$
\begin{aligned}
\Delta K+12 m(u)(\ln P)_{, u} & =0, \\
-2 m(u)_{, u} & =\kappa n^{2}(u) .
\end{aligned}
$$

In fact, it was demonstrated in [34] that such a separation can always be achieved using the coordinate freedom. It is then possible to reformulate equation (3) by introducing a $u$-dependent family of smooth 2-metrics $g_{a b}$ on the submanifold $r=$ const, $u=$ const, such that $g_{a b}=f(u, \zeta, \bar{\zeta})^{-2} g_{a b}^{0}$, where $g_{a b}^{0}(\zeta, \bar{\zeta})$ is the metric on a 2-dimensional sphere $S^{2}$. Since $g_{a b}$ is of the form $2 P^{-2} \mathrm{~d} \zeta \mathrm{d} \bar{\zeta}$ in our case, we can write

$$
P=f P_{0}, \quad P_{0}=1+\frac{1}{2} \zeta \bar{\zeta},
$$

and equation (3) becomes

$$
\frac{\partial f}{\partial u}=-\frac{1}{12 m(u)} f \Delta K
$$

where $\Delta$ is the Laplace operator associated with the metric $g_{a b}$. Denoting $\Delta_{0}$ and $K_{0}=1$ as the corresponding quantities related to $g_{a b}^{0}$, we obtain

$$
\Delta=f^{2} \Delta_{0}, \quad K=f^{2}\left(1+\Delta_{0}(\ln f)\right) .
$$




\section{Linear mass function}

Let us first consider the simplest choice of $m(u)$ which, in fact, has been widely used in literature (see e.g. $[7,9,36]$ ): we will assume that the mass function is a linearly decreasing positive function

$$
m(u)=-\mu u, \quad \mu=\text { const }>0,
$$

on the interval $\left[u_{0}, 0\right]$. Notice that for (8) the pure radiation field is uniform because equation (41) implies $n=\sqrt{2 \mu / \kappa}=$ const, independent of the retarded time $u$. The constant value $u_{0}<0$ localises an initial null hypersurface (that extends between the curvature singularity at $r=0$ and the conformal infinity $r=\infty$ ) on which an arbitrary sufficiently smooth initial data given by the function

$$
f_{0}(\zeta, \bar{\zeta})=f\left(u=u_{0}, \zeta, \bar{\zeta}\right)
$$

are prescribed, see Fig. 11.

\subsection{Existence of the solutions}

Now, the idea is to employ the Chruściel-Singleton results [29-31] concerning the analysis of the Robinson-Trautman vacuum equation, in particular the existence and asymptotic behaviour of its solutions. In the vacuum case $m$ in equation (3) is constant, and the solution $f(u, \zeta, \bar{\zeta})$ of the characteristic initial value problem (9) exists and is unique (in spite of the singularity at $r=0$ ). In the presence of pure radiation given by (8) it is possible to "eliminate" the variable mass function from the Robinson-Trautman field equation (6) mathematically by a simple reparametrisation

$$
\tilde{u}=-\mu^{-1} \ln (-u)
$$

cf. [34]. Indeed, equation (66) is then converted to

$$
\frac{\partial \tilde{f}}{\partial \tilde{u}}=-\frac{1}{12} \tilde{f} \tilde{\Delta} \tilde{K}
$$

where $\tilde{f}(\tilde{u}, \zeta, \bar{\zeta})=f(u(\tilde{u}), \zeta, \bar{\zeta}), \tilde{K}=\tilde{f}^{2}\left(1+\Delta_{0} \ln (\tilde{f})\right)$, and $\tilde{\Delta}=\tilde{f}^{2} \Delta_{0}$. Notice that the transformation (101) moves the hypersurface $u=0$, on which the mass function $m(u)$ reaches zero, to $\tilde{u}=+\infty$.

Chruściel [30] derived the following asymptotic expansion (as $\tilde{u} \rightarrow \infty$ ) for the function $\tilde{f}$ satisfying the evolution equation (11) for any smooth initial data $\tilde{f}_{0}=f_{0}$ on $\tilde{u}_{0}=-\mu^{-1} \ln \left(-u_{0}\right)$, namely

$$
\begin{aligned}
\tilde{f}= & 1+f_{1,0} e^{-2 \tilde{u}}+f_{2,0} e^{-4 \tilde{u}}+\cdots+f_{14,0} e^{-28 \tilde{u}} \\
& +f_{15,1} \tilde{u} e^{-30 \tilde{u}}+f_{15,0} e^{-30 \tilde{u}}+\cdots \\
= & \sum_{i=0}^{\infty} \sum_{j=0}^{N_{i}} f_{i, j} \tilde{u}^{j} e^{-2 i \tilde{u}}
\end{aligned}
$$

where $f_{i, j}$ are smooth functions on $S^{2}$ such that $f_{i, j}=0$ for $j>0, i \leq 14$. The function $\tilde{f}$ thus exists and converges exponentially fast to 1 , which means physically that the radiative Robinson-Trautman vacuum spacetimes approach asymptotically the Schwarzschild(anti-)de Sitter solution as $\tilde{u} \rightarrow \infty$, see relation (5). In our case of pure radiation field (8) 
we employ the transformation (10) on expression (12) to obtain the following asymptotic expansion of $f$ as $u \rightarrow 0_{-}$,

$$
\begin{aligned}
f= & 1+f_{1,0}(-u)^{2 / \mu}+f_{2,0}(-u)^{4 / \mu}+\cdots+f_{14,0}(-u)^{28 / \mu} \\
& -\mu^{-1} f_{15,1} \ln (-u)(-u)^{30 / \mu}+f_{15,0}(-u)^{30 / \mu}+\cdots \\
= & \sum_{i=0}^{\infty} \sum_{j=0}^{N_{i}} f_{i, j}\left[-\mu^{-1} \ln (-u)\right]^{j}(-u)^{2 i / \mu}
\end{aligned}
$$

As a result, for the initial data (9) the Robinson-Trautman type II spacetimes which contain uniform pure radiation field with the linear mass function (8) do exist in the whole region $u_{0} \leq u<0$. It is also obvious that the function $f$ approaches 1 as $u \rightarrow 0_{-}$ (where also $m(u) \rightarrow 0$ ) according to (13). In other words, these spacetimes approach the spherically symmetric Vaidya-(anti-)de Sitter metric near $u=0$.

The global structure of such spacetimes is schematically indicated on Fig. 1. In fact, there are two possibly different conformal diagrams depending on the value of $\mu$ : for $\mu>1 / 16$ there is a white hole singularity at $r=0$, for $\mu \leq 1 / 16$ there is also a naked singularity, see e.g. $[9,13,16,36]$ for more details. At $u=0$ all of the mass $m(u)$ is radiated away, and we can attach Minkowski space (de Sitter space when $\Lambda>0$, anti-de Sitter when $\Lambda<0$; the presence of the cosmological constant would change the character of conformal infinity $\mathcal{I}$ which would become spacelike or timelike, respectively) in the region $u>0$ along the hypersurface $u=0$. We will now investigate the smoothness of such an extension.

\subsection{Extension of the metric across $u=0$}

It follows from (13) that the smoothness of $f$ on $u=0$ is only finite. Depending on the value of $\mu$ two different cases have to be discussed separately: $2 / \mu$ is an integer, and $2 / \mu$ is a real non-integer positive number.

When $2 / \mu$ is an integer then due to the presence of the $\ln (-u)$ term associated with $f_{15,1} \neq 0$ the function $f$ is of the class $C^{(30 / \mu)-1}$. For $\mu$ very small, the integer number $(30 / \mu)-1$ is large so that $f$ becomes smoothly extendable to 1 across $u=0$ as $\mu \rightarrow 0$. This represents a naked-singularity Robinson-Trautman spacetime (see the right part of Fig. (1) unless $\mu=0$ which gives flat space everywhere. In the limiting case $\mu=1 / 16$ the function $f$ is of the class $C^{479}$. For the (white hole) Robinson-Trautman spacetimes given by $\mu>1 / 16$ the smoothness is lower. However, it is always at least $C^{14}$ because $\mu \leq 2$ in this case.

In the generic case when $2 / \mu$ is not an integer the function $f$ is only of the class $C^{\{2 / \mu\}}$, where the symbol $\{x\}$ denotes the largest integer smaller than $x$. Again, with $\mu \rightarrow 0$ the function $f$ becomes smoothly extendable. For $\mu<1 / 16$ the function $f$ is at least of the class $C^{32}$, for $\mu>2$ it is not even $C^{1}$ but it remains continuous.

To investigate further the smoothness of the metric when approaching the hypersurface $u=0_{-}$which is the analogue of the Schmidt-Tod boundary of vacuum RobinsonTrautman spacetimes $[25,30]$ we should consider the conformal picture using suitable double-null coordinates. Such Kruskal-type coordinates for the Vaidya solution with linear mass function (8) were introduced by Hiscock [7-9], see also [16,36], and we will use this transformation only to replace the coordinate $r$ since the null coordinate $u$ is already appropriate. Introducing a new coordinate $w$ by

$$
\mathrm{d} w=\frac{\mathrm{d} u}{u}-\frac{2 \mathrm{~d} z}{z\left(2 \mu z^{2}-z+2\right)}, \quad \text { where } \quad z=-\frac{u}{r}
$$


we put the Robinson-Trautman metric with linear mass function into the form

$$
\begin{aligned}
\mathrm{d} s^{2} & =-\left(K-1-2 \frac{f_{, u}}{f} r\right) \mathrm{d} u^{2} \\
& -\left(2 r+u+2 \mu \frac{u^{2}}{r}\right) \mathrm{d} u \mathrm{~d} w+2 \frac{r^{2}}{P^{2}} \mathrm{~d} \zeta \mathrm{d} \bar{\zeta}
\end{aligned}
$$

where $r(u, w)$. For the pure Vaidya metric characterized by $f=1$ and $K_{0}=1$ the first term vanishes identically so that the coordinates of (15) are indeed the Kruskal-type coordinates for the Vaidya spacetime with a linear mass function.

The smoothness of a general Robinson-Trautman metric (15) depends only on the smoothness of the metric coefficients $g_{u u}$ and $g_{\zeta \bar{\zeta}}$ (containing the function $f$ ) since the coefficient $g_{u w}$ tends to $-r$ as $u \rightarrow 0$. The smoothness of $g_{\zeta \bar{\zeta}}$ (for any finite $r$ ) and of $K$ is the same as of $f$, see (7). The function $f_{, u} / f$ is evidently one order less smooth than $f$. Consequently, for $2 / \mu$ being integer or non-integer number, the metric (15) is of the class $C^{(30 / \mu)-2}$ or $C^{\{2 / \mu\}-1}$, respectively. We again observe that the spacetimes approaching the linear Vaidya metric with naked singularity (i.e., for small values of the parameter $\mu$ ) possess higher order of smoothness at $u=0$.

One might be worried about the invariance of our results, namely with respect to a rescaling of the null coordinate $u(\hat{u})$ leading to a different smoothness of the function $f$ and of the metric. In order to change the smoothness on the hypersurface $u=0$ such rescaling must have a singular character there. But this would lead to a degeneracy of the metric coefficient $g_{\hat{u} w}$ of the Vaidya metric, which is forbidden. Consequently, the above results are in this sense unique.

We would like to obtain analogous results concerning smoothness of the extension also for a non-zero value of the cosmological constant $\Lambda$. Unfortunately, as far as we know, there is no explicit transformation of the Vaidya-de Sitter metric to the Kruskal-type coordinates even for the linear mass function (contrary to the Schwarzschild-de Sitter case [33]). However, it is possible to start with the Vaidya-de Sitter metric

$$
\mathrm{d} s^{2}=-h(u, r) \mathrm{d} u^{2}-2 \mathrm{~d} u \mathrm{~d} r+r^{2} \mathrm{~d} \Omega^{2}
$$

where $h(u, r)=1+2 \mu u r^{-1}-\frac{\Lambda}{3} r^{2}$, and perform a coordinate transformation

$$
\mathrm{d} w=g \mathrm{~d} u+2 \frac{g}{h} \mathrm{~d} r
$$

where $g(u, r)$ is some function. We arrive at the double-null form for the metric

$$
\mathrm{d} s^{2}=-\frac{h}{g} \mathrm{~d} u \mathrm{~d} w+r^{2}(u, w) \mathrm{d} \Omega^{2} .
$$

Of course, we have to ensure that $\mathrm{d} w$ in (17) is a differential of the coordinate $w$. The integrability condition $\left(\mathrm{d}^{2} w=0\right)$ gives the following quasilinear PDE,

$$
h^{2} \frac{\partial g}{\partial r}-2 h \frac{\partial g}{\partial u}+4 \frac{\mu}{r} g=0
$$

for the undetermined function $g$, which is difficult to solve analytically. The method of characteristic curves leads to the first-order ODE of the Abel type which has not yet been solved, but the existence of its solution is guaranteed. [It is possible to apply the perturbative approach starting from the solvable case of the de Sitter metric $(\mu=0)$ and 
then linearise the PDE in the parameter $\mu$. The result, however, can not be presented in a useful closed form.] For our purposes it suffices to use a general argumentation: the coordinate $u$ is already suitably compactified and we are only determining the complementary null coordinate $w$ to obtain the Vaidya-de Sitter metric in the Kruskal-type coordinates (which is smooth on $u=0$ ). The corresponding Robinson-Trautman metric in these coordinates differs only by the term $g_{u u}(u, r, \zeta, \bar{\zeta}) \mathrm{d} u^{2}$ (which is absent in the Vaidya-de Sitter case in the double null coordinates), and by a different metric coefficient $g_{\zeta \bar{\zeta}}=r^{2} f^{-2} P_{0}^{-2}$, where $r(u, w)$ is finite and smooth when approaching the hypersurface $u=0$. The smoothness is thus not affected by the specific transformation (17) and it is the same as for the vanishing cosmological constant. This is different from vacuum spacetimes with $m=$ const $\neq 0$ studied in $[32,33]$ because in the present case $m \rightarrow 0$ near $u=0$, and the influence of $\Lambda$ on the smoothness becomes negligible.

\section{General mass function}

The results obtained above can be considerably generalized. Inspired by a similar idea outlined in [34] we may consider a reparametrisation on the null coordinate $u$ by

$$
\tilde{u}=\gamma(u)
$$

where $\gamma$ is an arbitrary continuous strictly monotonous function. We start with the evolution equation (11) for which the existence and uniqueness of solutions has been proven, and their general asymptotic behaviour (12) has been demonstrated. Now, by applying the substitution (20) in equation (11) we obtain

$$
\frac{\partial f}{\partial u}=-\frac{\dot{\gamma}}{12} f \Delta K
$$

(where the dot denotes a differentiation) which is the evolution equation for the function $f(u, \zeta, \bar{\zeta})$. This is exactly the Robinson-Trautman equation (6) for the mass function

$$
m(u)=\frac{1}{\dot{\gamma}(u)} .
$$

For a given smooth initial data on $u_{0}$ there thus exists the Robinson-Trautman spacetime (11), including the cosmological constant $\Lambda$, with the mass function (22). To obtain a positive mass we consider a growing function $\gamma(u)$. Considering (4) this corresponds to a universe filled with homogeneous pure radiation

$$
n^{2}(u)=\frac{2}{\kappa} \frac{\ddot{\gamma}}{\dot{\gamma}^{2}}
$$

For consistency the function $\gamma$ must be convex. An asymptotic behaviour of the function $f$ as $\gamma(u) \rightarrow \infty$ is easily obtained from the expansion (12) by substituting relation (20).

In particular, the linear mass function (8) discussed above is a special case of (22) for the transformation (20) of the form (10). More general explicit solutions can be obtained, e.g., by considering the power function

$$
\gamma(u)=(-u)^{-p}, \quad p>0
$$

which gives

$$
m(u)=\frac{1}{p}(-u)^{1+p}, \quad n^{2}(u)=\frac{2(p+1)}{\kappa p}(-u)^{p}
$$


Both functions $m$ and $n$ approach zero as $u \rightarrow 0$. Due to the theorems mentioned above, there exist Robinson-Trautman type II spacetimes in the region $u<0$ which approach the spherically symmetric Vaidya-(anti-)de Sitter metric as $u \rightarrow 0_{-}$with the mass function and pure radiation given by (25). The asymptotic behaviour of such solutions is determined by expression (51) with

$$
f=1+\sum_{i=1}^{\infty} \sum_{j=0}^{N_{i}} f_{i, j}(-u)^{-j p} \exp \left[-2 i(-u)^{-p}\right]
$$

where $f_{i, j}=0$ for $j>0$ if $i \leq 14$. Interestingly, the function $f$ is now smooth on $u=0$ for any power coefficient $p$, but this still does not guarantee that the extension into flat region $u>0$ is analytic (see [33] for a similar situation).

Another simple explicit choice is

$$
\gamma(u)=-M^{-1} \ln [\sinh (-u)], \quad M>0,
$$

which implies (see also [16])

$$
m(u)=M \tanh (-u), \quad n^{2}(u)=\frac{2 M}{\kappa \cosh ^{2} u} .
$$

In the region $u<0$ the mass function monotonically decreases from $M$ to zero, while the pure radiation field grows from zero to the value $2 M / \kappa$ as $u \rightarrow 0$. Let us note that in this case the integrated radiation density is finite on the interval $(-\infty, 0), \int_{-\infty}^{0} n^{2}(u)=2 M / \kappa$. The expansion near $u=0_{-}$is

$$
f=1+\sum_{i=1}^{\infty} \sum_{j=0}^{N_{i}} f_{i, j}\left(-M^{-1} \ln [\sinh (-u)]\right)^{j} \sinh ^{2 i / M}(-u) .
$$

If $2 / M$ is an integer then the function $f$ belongs to the class $C^{(30 / M)-1}$, otherwise it is of the class $C^{\{2 / M\}}$.

\section{Possible modifications and applications}

The Robinson-Trautman pure radiation solutions in the region $u_{0} \leq u \leq 0$ approaching the Vaidya metric near $u=0$, which can be extended (albeit non-smoothly) to flat Minkowski space in the region $u \geq 0$ as in Fig. 1 may be used for construction of various models of radiative spacetimes. For example, it is natural to further extend the solution "backwards" into the region $u_{1}<u \leq u_{0}$ by the Robinson-Trautman vacuum solution with a constant mass $m_{0}=m\left(u_{0}\right)$, such that the function $f$ is continuous on $u_{0}$. This is shown in Fig. 2. In such a case the spacetime may describe the process of "evaporation" of a white hole (with a different character of the singularity at $r=0$ when $\mu \leq 1 / 16$ ) with its mass decreasing from the value $m_{0}$ to zero. Let us emphasize that the region $u<u_{0}$ does not represent the Schwarzschild solution because the spacetime is not spherically symmetric there $(f \neq 1)$. In fact, this is the region where the original Chruściel theorems on the behaviour of the Robinson-Trautman vacuum spacetimes with constant mass apply (cf. (11), (12)). However, the spacetime in this region can not be extended up to the past conformal infinity $\mathcal{I}^{-}$because the metric function $f$ diverges as $u \rightarrow-\infty$.

In the presence of the cosmological constant $\Lambda$ one obtains a family of exact spacetimes that describe evaporation of a white hole in the (anti-)de Sitter universe. In this case the 

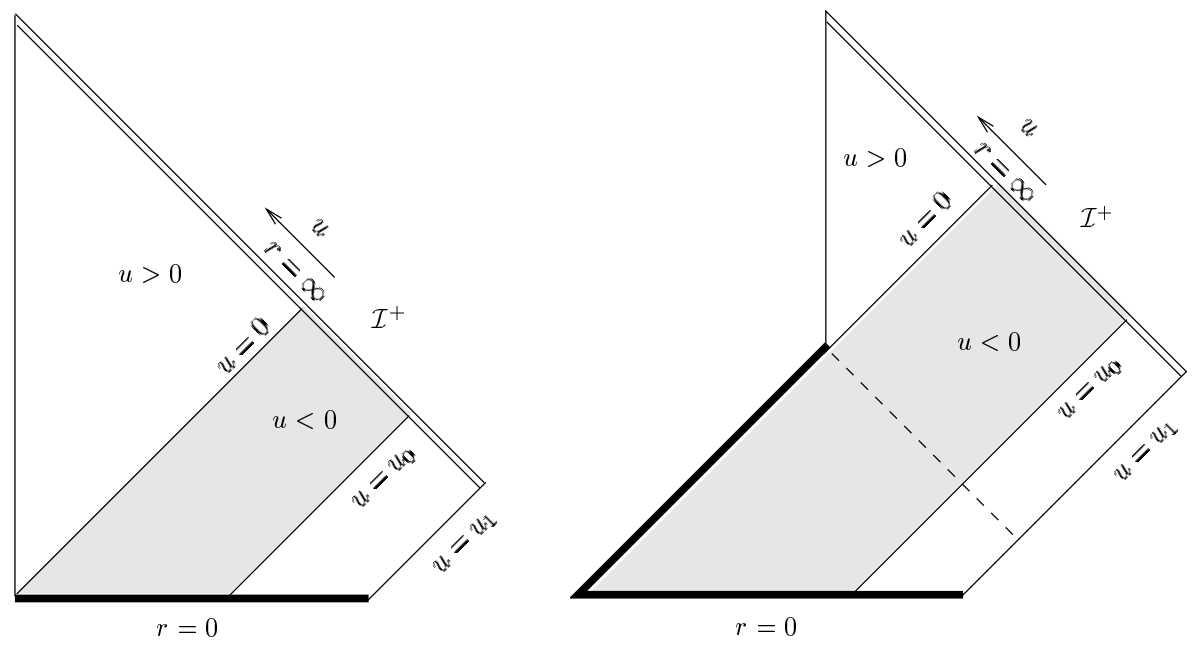

Figure 2: Possible extensions of the Robinson-Trautman radiative spacetimes into the region $u<u_{0}$. Pure radiation is present only in the shaded region, everywhere else it is a vacuum solution. For $u \in\left(u_{1}, u_{0}\right)$ the mass function is constant, $m\left(u_{0}\right)=-\mu u_{0}$, but the spacetime is not spherically symmetric - it is not the Schwarzschild solution $(\mu>1 / 16$ on the left, $\mu \leq 1 / 16$ on the right).

schematic conformal diagram on Fig. 2 has to be modified in such a way that for all values of $u$ the conformal infinity $\mathcal{I}^{+}$becomes timelike (for $\Lambda>0$ ) or spacelike (for $\Lambda<0$ ).

Another possible modification is to consider the "advanced" form of the spacetimes (which describes an ingoing flow) rather than the "retarded" form (corresponding to outgoing flow) employed above (see, e.g., [13] for more details). This time-reversed form is obtained formally by a simple substitution $u \rightarrow-v$ in the metrics and corresponding functions. The Robinson-Trautman metric thus reads

$$
\mathrm{d} s^{2}=-\left(K+2 r(\ln P)_{, v}-2 \frac{m}{r}-\frac{\Lambda}{3} r^{2}\right) \mathrm{d} v^{2}+2 \mathrm{~d} v \mathrm{~d} r+2 \frac{r^{2}}{P^{2}} \mathrm{~d} \zeta \mathrm{d} \bar{\zeta},
$$

where $m(v)$ is an increasing mass function in $v \in\left[0, v_{0}\right]$. This is joined with flat Minkowskian region $v<0$, and extended to the region $v \geq v_{0}$ by the corresponding Robinson-Trautman(anti-)de Sitter black hole vacuum solution, see Fig. 3. It is a non-spherical generalization of the gravitational collapse of a shell of null dust forming a naked singularity $[9,37,38]$ in these works the mass function was taken to be $m(v)=\mu v$ (with $m(v)=0$ for $v \leq 0$, and $m(v)=M=\mu v_{0}$ for $\left.v \geq v_{0}\right)$. The metric function $P$ is now given by $P=f P_{0}$ where $f$ is analogous to (13),

$$
f=\sum_{i=0}^{\infty} \sum_{j=0}^{N_{i}} f_{i, j}\left(-\mu^{-1} \ln v\right)^{j} v^{2 i / \mu},
$$

so that the smoothness of the metric on the boundary $v=0$ depends on the parameter $\mu$. For $v \in\left(v_{0}, v_{1}\right)$ the spacetime is vacuum but not spherically symmetric. The metric diverges as $v \rightarrow \infty$. Our results can thus be interpreted in such a way that - at least within the Robinson-Trautman family of solutions - the model [9] of collapse to a naked shell-focusing singularity which is based on the spherically symmetric Vaidya metric is not stable against perturbations. 

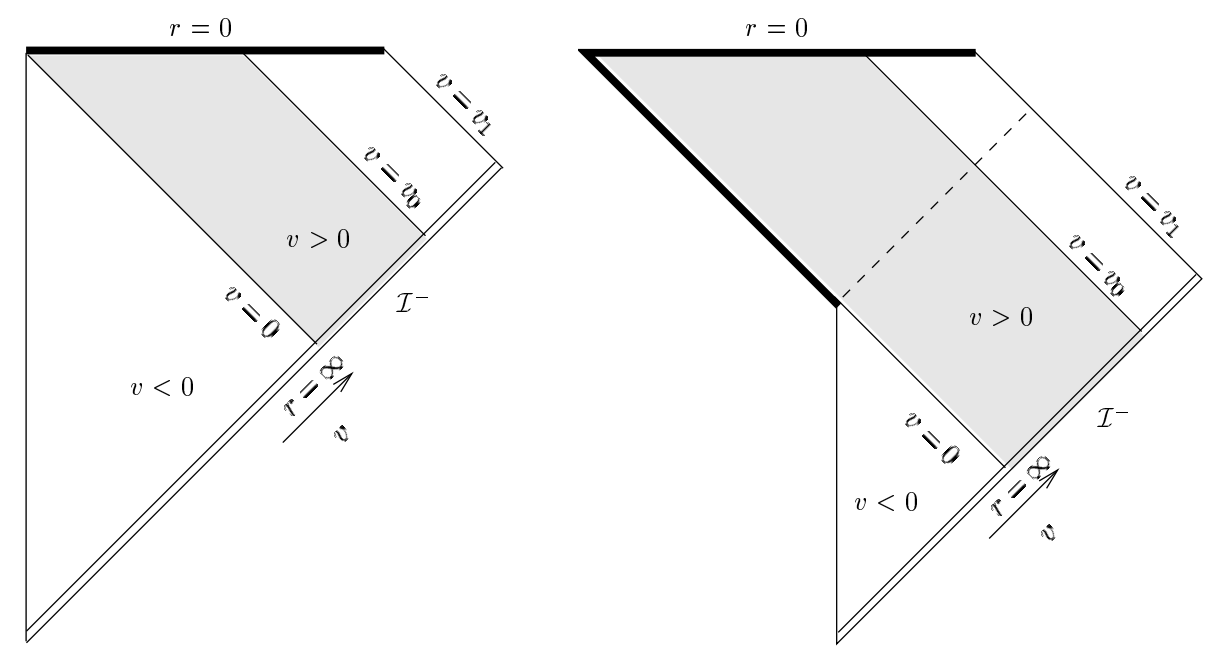

Figure 3: Time-reversed version of Fig. 2 represents the "advanced" form of the RobinsonTrautman spacetimes (30) which describes an ingoing flow of radiation.

\section{Concluding remarks}

In our contribution we have analyzed exact solutions of the Robinson-Trautman class which contain homogeneous pure radiation and a cosmological constant. This is a natural extension of previous works [19-34] on properties of vacuum spacetimes of this family. We have demonstrated that these solutions exist for any smooth initial data, and that they approach the spherically symmetric Vaidya-(anti-)de Sitter metric. It generalizes previous results according to which vacuum Robinson-Trautman spacetimes approach asymptotically the spherically symmetric Schwarzschild-(anti-)de Sitter metric. We have investigated extensions of these solutions into Minkowski region, and we have shown that its order of smoothness is in general only finite. Finally, we suggested some applications of the results. For example, it follows that the model of gravitational collapse of a shell of null dust diverges as $v \rightarrow \infty$ which indicates that investigations of such process based on the spherically symmetric Vaidya metric are, in fact, not stable against "non-linear perturbations", at least within the Robinson-Trautman family of exact solutions.

\section{Acknowledgements}

We are grateful to Jiří Bičák for valuable comments, and Jerry Griffiths for reading the manuscript.

\section{References}

[1] P. C. Vaidya, Current Science 12, 183 (1943).

[2] P. C. Vaidya, Proc. Indian Acad. Sci. A 33, 264 (1951).

[3] P. C. Vaidya, Nature 171, 260 (1953). 
[4] H. Stephani, D. Kramer, M. MacCallum, C. Hoenselaers, and E. Herlt, Exact Solutions of Einstein's Field Equations, Second Edition (Cambridge University Press, Cambridge, England, 2002)

[5] A. Wang and Y. Wu, Gen. Relativ. Gravit. 31, 107 (1999).

[6] A. Krasiński, Gen. Relativ. Gravit. 31, 115 (1999).

[7] W. A. Hiscock, Phys. Rev. D 23, 2813 (1981).

[8] W. A. Hiscock, Phys. Rev. D 23, 2823 (1981).

[9] W. A. Hiscock, L. G. Williams, and D. M. Eardley, Phys. Rev. D 26, 751 (1982).

[10] Y. Kuroda, Prog. Theor. Phys. 71, 100 (1984).

[11] Y. Kuroda, Prog. Theor. Phys. 71, 1422 (1984).

[12] J. Bičák and K. V. Kuchař, Phys. Rev. D 56, 4878 (1997).

[13] J. Bičák and P. Hájičček, Phys. Rev. D 68, 104016 (2003).

[14] S. G. Ghosh and N. Dadhich, Phys. Rev. D 64, 047501 (2001).

[15] T. Harko, Phys. Rev. D 68, 064005 (2003).

[16] F. Girotto and A. Saa, Phys. Rev. D 70, 084014 (2004).

[17] I. Robinson and A. Trautman, Phys. Rev. Lett. 4, 431 (1960).

[18] I. Robinson and A. Trautman, Proc. Roy. Soc. Lond. A265, 463 (1962).

[19] J. Foster and E. T. Newman, J. Math. Phys. 8, 189 (1967).

[20] B. Lukács, Z. Perjés, J. Porter, and Á. Sebestyén, Gen. Relativ. Gravit. 16, 691 (1984).

[21] M. A. J. Vandyck, Class. Quantum Grav. 2, 77 (1985).

[22] M. A. J. Vandyck, Class. Quantum Grav. 4, 759 (1987).

[23] B. G. Schmidt, Gen. Relativ. Gravit. 20, 65 (1988).

[24] A. D. Rendall, Class. Quantum Grav. 5, 1339 (1988).

[25] K. P. Tod, Class. Quantum Grav. 6, 1159 (1989).

[26] E. W. M. Chow and A. W. C. Lun, J. Austr. Math. Soc. B 41, 217 (1999).

[27] D. B. Singleton, Class. Quantum Grav. 7, 1333 (1990).

[28] S. Frittelli and O. M. Moreschi, Gen. Relativ. Gravit. 24, 575 (1992).

[29] P. T. Chruściel, Commun. Math. Phys. 137, 289 (1991).

[30] P. T. Chruściel, Proc. Roy. Soc. Lond. A436, 299 (1992).

[31] P. T. Chruściel and D. B. Singleton, Commun. Math. Phys. 147, 137 (1992). 
[32] J. Bičák and J. Podolský, Phys. Rev. D 52, 887 (1995).

[33] J. Bičák and J. Podolský, Phys. Rev. D 55, 1985 (1997).

[34] J. Bičák and Z. Perjés, Class. Quantum Grav. 4, 595 (1987).

[35] J. Bičák and J. Podolský, J. Math. Phys. 40, 4495 (1999).

[36] B. Waugh and K. Lake, Phys. Rev. D 34, 2978 (1986).

[37] I. H. Dwivedi and P. S. Joshi, Class. Quantum Grav. 6, 1599 (1989).

[38] I. H. Dwivedi and P. S. Joshi, Class. Quantum Grav. 8, 1339 (1991). 